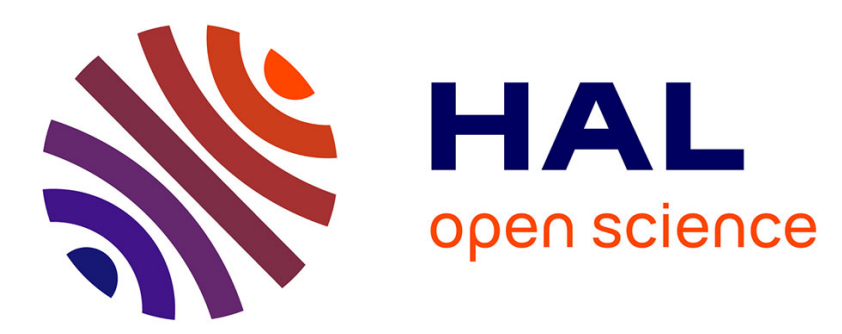

\title{
Using a Modified Taylor Cell to Validate Simulation and Measurement of Field-to-Shorted-Trace Coupling
}

\author{
Sjoerd Op 'T Land, Mohamed Ramdani, Richard Perdriau, Yannis Braux, \\ M'Hamed Drissi
}

\section{- To cite this version:}

Sjoerd Op 'T Land, Mohamed Ramdani, Richard Perdriau, Yannis Braux, M'Hamed Drissi. Using a Modified Taylor Cell to Validate Simulation and Measurement of Field-to-Shorted-Trace Coupling. IEEE Transactions on Electromagnetic Compatibility, 2014, 56 (4), pp.864-870. 10.1109/TEMC.2014.2313231 . hal-01087147v2

\section{HAL Id: hal-01087147 \\ https://hal.inria.fr/hal-01087147v2}

Submitted on 26 Nov 2014

HAL is a multi-disciplinary open access archive for the deposit and dissemination of scientific research documents, whether they are published or not. The documents may come from teaching and research institutions in France or abroad, or from public or private research centers.
L'archive ouverte pluridisciplinaire HAL, est destinée au dépôt et à la diffusion de documents scientifiques de niveau recherche, publiés ou non, émanant des établissements d'enseignement et de recherche français ou étrangers, des laboratoires publics ou privés.

$$
\text { Copyright }
$$




\title{
Using a Modified Taylor Cell to Validate Simulation and Measurement of Field-to-Shorted-Trace Coupling
}

\author{
Sjoerd Op 't Land*, Student Member, IEEE, Mohamed Ramdani*, Senior Member, IEEE, \\ Richard Perdriau*, Senior Member, IEEE, Yannis Braux ${ }^{\dagger}, \mathrm{M}^{\prime}$ hamed Drissi ${ }^{\ddagger}$, Senior Member, IEEE \\ *ESEO-IETR \\ Angers, France \\ Email: \{sjoerd.optland, richard.perdriau, mohamed.ramdani\}@eseo.fr \\ CST-Computer Simulation Technology AG \\ Darmstadt, Germany \\ Email: yannis.braux@cst.com \\ †Université européenne de Bretagne IETR \\ Rennes, France \\ Email: mhamed.drissi@insa-rennes.fr
}

\begin{abstract}
Predicting the immunity of electronic boards to radiated electromagnetic interference requires the computation of the coupling efficiency of an electromagnetic field to PCB traces. In the case of complex PCBs, full-wave electromagnetic solvers are convenient, yet at the expense of simulation time. Therefore, this paper introduces the extension of a modified Taylor-based analytical model to the case of traces terminated at one end by a non-characteristic impedance. This model makes it possible to determine the far-field-to-trace coupling using only a sum of closed-form equations. When applied to a shorted, meandered PCB trace, it was found to be as precise as $2.2 \mathrm{~dB}$ average absolute error with respect to GTEM measurements, which demonstrates its relevance for immunity prediction. Moreover, the full-wave simulation of this case study was validated using the extended model and found to be as precise as $1.4 \mathrm{~dB}$ average absolute error.

Index Terms-field-to-trace coupling, modified Taylor model, closed-form solution, full-wave simulation, GTEM cell
\end{abstract}

\section{INTRODUCTION}

Electromagnetic compatibility (EMC) problems can be understood as a three-element chain: source-transfer pathreceiver [1]. In the case of unshielded, wireless electronics, the dominant transfer path can consist in PCB traces. In that case, the routing of PCB traces is decisive for product compliance. Therefore, quickly estimating the field-to-trace coupling may be interesting.

Full-wave electromagnetic solvers can calculate this coupling for arbitrary trace geometry, terminal impedances, frequency, polarization and angle of incidence. However, these parameters need to be swept to find the average or worst case, which is time-consuming.

Therefore, other simplifications for this multi-dimensional problem have been discussed in the literature. Lagos developed an algorithm to find the worst case incidence for a straight trace with arbitrary terminal impedances and one frequency [2]. Magdowski found closed-form equations for the average coupling under random illumination to a straight two-wire transmission line with arbitrary terminal impedances [3]. Leone noticed that the coupling to matched terminal impedances is a reasonable approximation for moderate mismatches in terminal impedances [4].

Recently, a simple model was developed by the authors for a grazing, horrizonatly polarized incidence on a trace with matched terminal impedances: the modified Taylor cell [5], [6], which has a closed-form solution.

This paper introduces an improvement over this model for one mismatched terminal impedance. This model is then crosscompared with full-wave simulations and GTEM measurements on a realistic case study.

The paper is organized as follows: the aforementioned case study is first introduced in Section II: a meandered, shorted PCB trace illuminated by a grazing plane wave. The modified Taylor cell is then recalled in Section III. This model is then extended to include one non-characteristic load and is applied to the case study in Section IV. For reference, a fullwave electromagnetic simulation is set-up and applied to the case study in Section V. Finally, the experiment is performed in Section VI and compared with the full-wave solver and modified Taylor predictions. Conclusions are drawn and recommendations for future research are given in Section VII.

\section{Case Study: A Meandered, Shorted MicrostriP}

To allow for a meaningful comparison, a case study that can both be predicted theoretically and measured practically will be defined.

The theoretical prediction will be carried out both with modified Taylor cells and with a full-wave solver. As anything can be modeled in a full-wave solver, only the validity constraints of modified Taylor cells need to be considered. The modified Taylor cell predicts the coupling of a grazing, hofertically 


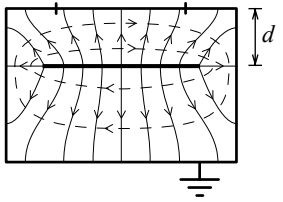

(a) GTEM frontal crosssection with electrostatic (solid, arrows) and magnetostatic (dashed) field lines.

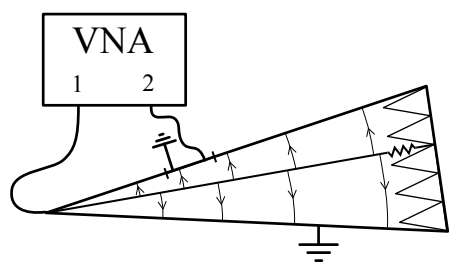

(b) Overview lateral cross-section with electrostatic field lines. The resistive/absorbent hybrid load provides broadband characteristic termination.

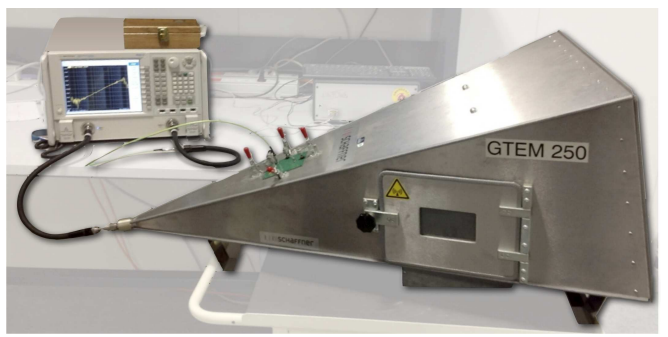

(c) Overview photo, relevant parts highlighted. polarized plane wave to one straight, characteristic microstrip segment [5]. If the impedance discontinuities between segments are negligible, a finite cascade of microstrip segments of different planar orientation can be modeled by cascading Taylor cells [6].

Practically, the GTEM-cell was chosen as the plane wave source. The GTEM-cell is basically a tapered rectangular, paraxial $50 \Omega$ waveguide with a metallic strip (the septum) as the center conductor, as schematized in Figure 1a. It sports a top aperture to illuminate a $10 \times 10 \mathrm{~cm}$ PCB. The waveguide has a coaxially connectorized input, and its other end is terminated with a DC-18 GHz load, thus minimizing standing waves in the waveguide. As a result, the field strength under the PCB can be approximated by dividing the septum voltage $V_{\text {septum }}$ by the septum distance $d$ [7].

To measure the broadband transfer from the GTEM-cell input to any trace terminal, a Vector Network Analyzer (VNA) is used. Consequently, any monitored terminal is necessarily loaded by $50 \Omega$. The resulting measurement set-up is shown in Figure 1.

The PCB needs to provide coaxial connectors to give access to both trace terminals. Given the available surface, a threesegment microstrip is chosen for simplicity's sake. Because the far end has the stronger high-frequency coupling for a straight microstrip [5], the far-end induced voltage is studied. At the near end, a short calibration standard is connected, inevitably including an electrical delay $\tau_{d}$. The entire path from connector to connector needs to be matched to the VNA characteristic impedance of $50 \Omega$. The resulting PCB and illumination are outlined in Figure 2.

\section{MODIFIED TAYLOR MODEL}

The analytical model used in this paper is based on Taylor's. This section recalls his model and the modification to take into account long line effects.

\section{A. Taylor's Model}

Taylor's model for field-to-line coupling represents the electric and magnetic induction as distributed sources along the transmission line. The specialization of the model for a twowire transmission line is shown in Figure 3. The component $H_{n}$ of the magnetic field (normal to the plane of the wires) induces a voltage, and the component $E_{t}$ of the electric field (in the plane and transversal to the wires) induces a current. Like a passive transmission line, each slice $\Delta z$ introduces a phase shift $\beta \Delta z$, where $\beta$ is the wavenumber or spatial frequency.

If frequency asymptotically tends to zero, the exciting wavelength is infinitely long. Since the line has finite length, in this case, the phase shift along the line tends to zero and the field can be considered spatially uniform. Consequently, the line can be lumped as a single cell $\Delta z=\ell$. In the case of characteristic loads $\left(R_{\mathrm{ne}}=R_{\mathrm{fe}}=Z_{c}\right)$ the low-frequency eitherend terminal voltages can be found by inspecting Figure $3 \mathrm{~b}$ [1]:

$$
V_{\mathrm{LF}}=-\frac{1}{2} \mathrm{j} \omega c E_{t} Z_{c} h \ell \mp \frac{1}{2} \mathrm{j} \omega \mu_{0} H_{n} h \ell,
$$

where $c$ is the per-unit-length (pul) capacitance of the line. Unless otherwise noted, the near-end and far-end results are presented simultaneously throughout the paper; $\mp$ means minus for the near end and plus for the far end.

\section{B. Modification for Long Lines}

If the field cannot be considered uniform along the line, the line needs to be lumped in infinitesimal slices $\mathrm{d} z$, along each of which the field can be considered uniform. The terminal voltages can then be found by summing the contributions of this infinity of cells. Consequently, the resulting equations are no longer in closed form, which is inconvenient.

Fortunately, it turns out that the closed-form single-cell model of (1) can be maintained with a simple modification. A simple demonstration will be given here; the reader can refer to [5] for the formal derivation.

The starting point of this demonstration is a thought experiment on the lossless, characteristically terminated line of

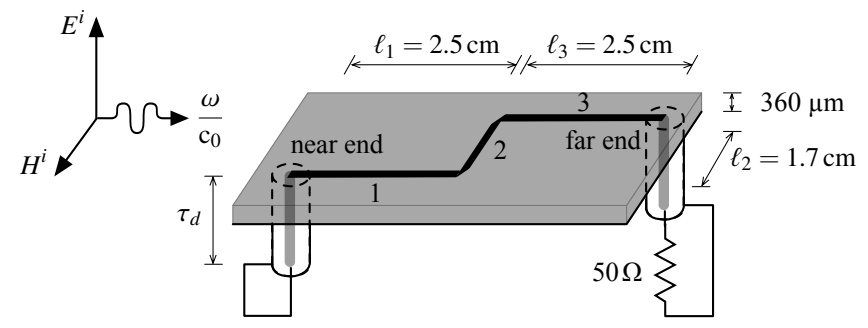

Figure 2. Perspective on this paper's case study PCB: a three-segmenty $50 \Omega$ microstrip trace over a ground plane, illuminated by a Korizontally polarized plane wave. The near end's coaxial connector is shorted, the far end's connector is terminated in a characteristic load (the VNA's input). 
Figure 3, illuminated from the near-end, that is, with the wave vector parallel to the line, pointing to the far end. The object of study is the far-end induced voltage, caused by a forward traveling wave on the line: the forward eigenwave. Its normalized amplitude $w$ is just a phase lag:

$$
w(z)=\mathrm{e}^{-\mathrm{j} \beta z} ; \quad \beta=\frac{\omega}{v},
$$

where $v$ is the phase speed of a wave on the transmission line. Likewise, the illuminating field has a normalized amplitude $i$ :

$$
i(z)=\mathrm{e}^{-\mathrm{j} k_{p} z} ; \quad k_{p}=\frac{\omega}{\mathrm{c}_{0}},
$$

where $k_{p}$ is the vector component of $k$ parallel to the line. Starting with an illumination frequency where the error introduced by only using a single Taylor cell is negligible, the following phenomena can be observed with increasing frequency along Figure 4(a-d).

Figure 4a shows the case for low frequencies, where the incident field remains the same along the line, so modeling the line as one Taylor cell is legitimate.

As shown in Figure 4b, the wavelength decreases. When the wavelength gets in the order of the line length, a propagating wave appears, both in free space and in the transmission line. Yet, this does not immediately invalidate the model. Indeed, the field is no longer uniform along the line, but the forward eigenwave of the line and the free space plane wave travel in the same direction. That means that, for every line slice, the free space wave and the eigenwave still have approximately the same phase. Therefore, it is still legitimate to model the line as one cell.

As frequency increases further, like in Figure 4c, the phase difference between the forward eigenwave and the incident plane wave becomes significant; in the example shown, the phase difference goes from 0 at $z=0$ to $\pi$ at $z=\ell$. On average, both waves are still cross-correlated, but less so than for low frequencies.

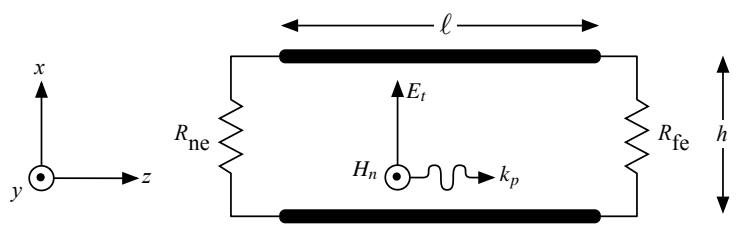

(a) Line geometry: subscripts $t, n$ and $p$ (or $x, y$ and $z$ ) denote excitation field components, respectively transversal, normal and parallel to the line segment. $R_{\mathrm{ne}}$ and $R_{\mathrm{fe}}$ are the near-end and far-end resistive terminations.

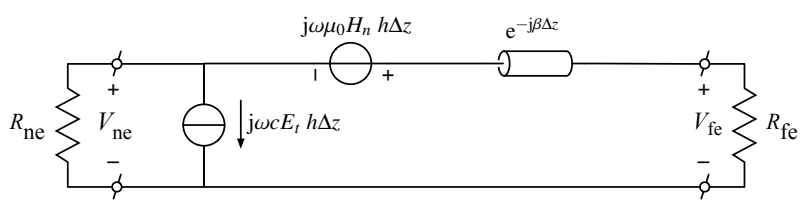

(b) Taylor's cell: approximation of passive transmission line slice $\Delta z$, with a voltage source representing the electromotive force (emf) and current source representing the electrostatic force (electric induction). $c$ denotes the per-unitlength (PUL) capacity of the line, $\beta$ is the wavenumber of the line.

Figure 3. Modeling the coupling of an electromagnetic wave to a two-wire transmission line.
In the extreme case of Figure $4 \mathrm{~d}$, the phase difference goes all the way from 0 at $z=0$ to $2 \pi$ at $z=\ell$. On average, the two waves are no longer cross-correlated and coupling is not expected any longer.

To summarize, a single Taylor cell correctly predicts coupling for low frequencies. However, it overestimates the coupling at high frequencies. The single Taylor cell can be corrected for high frequencies with a measure for the lengthaverage cross-correlation between the line's eigenwave and the incident wave. This measure should be unity for low frequencies, in order not to modify the low frequency coupling. This unitless measure $K$ should amount to zero when the phase difference along the line goes all the way from 0 to $2 \pi$.

The cross-correlation of the incident field and the line's eigenwave amplitudes is given by the complex conjugated product $i w^{*} . K$ is then found by averaging along the line:

$$
K=\frac{1}{\ell} \int_{0}^{\ell} i(z) \cdot w^{*}(z) \mathrm{d} z=\frac{1}{\mathrm{j}\left(-k_{p} \mp \beta\right) \ell}\left(\mathrm{e}^{\mathrm{j}\left(-k_{p} \mp \beta\right) \ell}-1\right) .
$$

To calculate the near-end induced voltage, the backward traveling eigenwave $w=\mathrm{e}^{+\mathrm{j} \beta z}$ is used. The resulting, modified Taylor cell is depicted in Figure 5.

\section{Application to Shorted and Meandered Trace}

These modified Taylor cells will now be applied to predict the coupling to the shorted and meandered trace.

\section{A. Near-End Non-Characteristic Load}

In the case where the illuminated line is not terminated in a characteristic load at the near end, with a characteristic load still present at the far end, the reflected near-end voltage now adds up to the far-end induced voltage, as outlined in Figure 6a. Because of the far-end characteristic load, there are

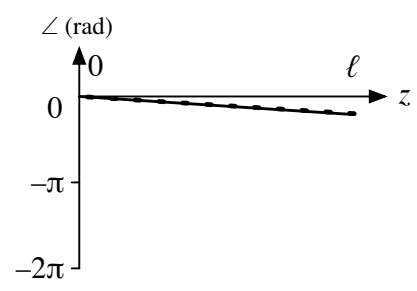

(a) Very low frequency.

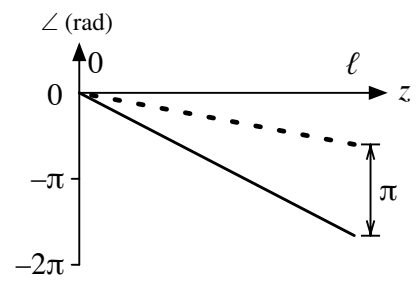

(c) High frequency.

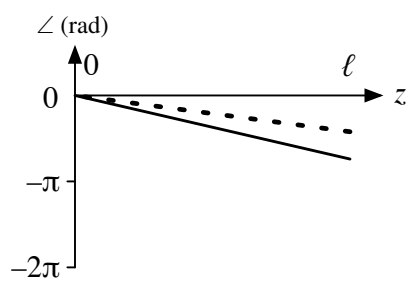

(b) Low frequency.

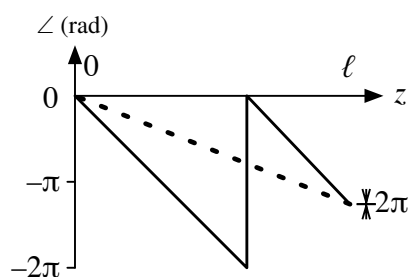

(d) Very high frequency.
Figure 4. Phase along the transmission line of the line's eigenwave $\angle w$ (solid line) and illuminating plane wave $\angle i$ (dashed line), for increasing frequency. 
no further reflections to consider. This translates to Figure $6 \mathrm{~b}$ and (5):

$$
\left.V_{\mathrm{fe}}\right|_{\Gamma_{\mathrm{ne}} \neq 0}=\left.V_{\mathrm{fe}}\right|_{\Gamma_{\mathrm{ne}=0}}+\left.\Gamma_{\mathrm{ne}} V_{\mathrm{ne}}\right|_{\Gamma_{\mathrm{ne}}=0} \mathrm{e}^{-\mathrm{j} \beta \ell},
$$

where $V_{\mathrm{fe}}$ is the far-end induced voltage and $\Gamma_{\mathrm{ne}}$ is the voltage reflection coefficient $\left(Z_{\mathrm{ne}}-Z_{c}\right) /\left(Z_{\mathrm{ne}}+Z_{c}\right)$ of the near-end terminating impedance $Z_{\text {ne }}$. For a single segment, the induced voltage can be found by inspecting Figure 5 with (4):

$$
\begin{aligned}
\left.V_{\mathrm{fe}}\right|_{\Gamma_{\mathrm{ne}} \neq 0}= & \frac{1}{2} \mathrm{j} \omega\left(+\mu_{0} H_{n}(0)-c E_{t}(0) Z_{c}\right) h \ell K_{\mathrm{fe}} \mathrm{e}^{-\mathrm{j} \beta \ell}+ \\
& \Gamma_{\mathrm{ne}} \frac{1}{2} \mathrm{j} \omega\left(-\mu_{0} H_{n}(0)-c E_{t}(0) Z_{c}\right) h \ell K_{\mathrm{ne}} \mathrm{e}^{-\mathrm{j} \beta \ell} .
\end{aligned}
$$

In the case study, the near-end impedance consists of a short circuit, preceded by a coaxial waveguide:

$$
\Gamma_{\mathrm{ne}}=-1 \mathrm{e}^{-\mathrm{j} \omega \cdot 2 \tau_{d}},
$$

where $\tau_{d}$ is the one-way electrical delay of the coaxial connector and in the connected short-circuit standard.

\section{B. Application to Meandered PCB Trace}

To apply this modified Taylor cell to the case study, the two-wire line must be transformed into a microstrip trace [5] and a single segment into three segments [6].

The essential difference between a two-wire line and a microstrip is the presence of a ground plane and a substrate.

The ground plane doubles the field strength $E^{i}$. This can be understood from the case of a Hertzian dipole, just above the $z y$-plane, infinitely far from the origin, such that the field $E_{x}$ is $1 \mathrm{~V} / \mathrm{m}$ at the origin. If a ground plane is now placed at the $z y$-plane, there will be no field anymore under the $z y$-plane and the field above it will have doubled. It must be noted that this free space field $2 E^{i}$ equals the parallel plate field $V_{\text {septum }} / d$ of the GTEM-cell.

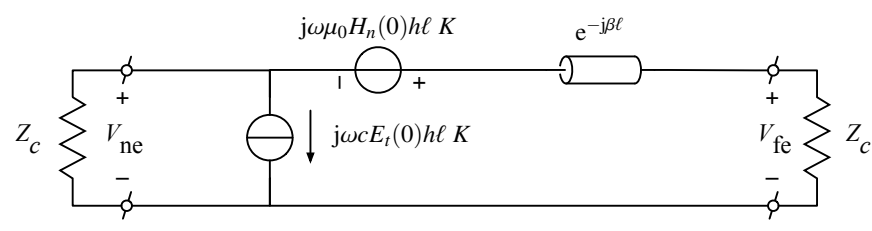

Figure 5. Modified Taylor cell taking into account long-line effects.
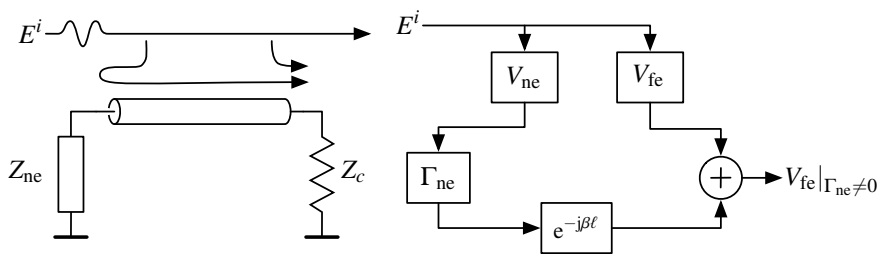

(a) Propagating waves: the illu- (b) Induced voltage calculation graph for minating wave induces a near- the far end. end and a far-end voltage.

Figure 6. Propagating waves and induced voltage calculation for the case of a non-characteristic near-end load and a characteristic far-end load.
As far as the dielectric substrate is concerned, the plane wave just above is imposed. Since the field in the substrate must follow with a constant phase lag, the wavenumber in the dielectric is equal to that in free space. Moreover, the material is not magnetic, hence the magnetic field $H$ in the substrate is $2 H^{i}$. Considering the substrate as part of an infinitely broad parallel-plate voltage divider, the electric field $E$ in the substrate turns out to be $2 E^{i} / \varepsilon_{r}$. These conclusions are summarized in Figure 7.

To model a three-segment microstrip, the contributions of the straight line segments $\ell_{1}, \ell_{2}$ and $\ell_{3}$ must be summed:

$$
\begin{aligned}
\left.V_{\mathrm{fe}}\right|_{\Gamma_{\mathrm{ne}}=0} & =\left(K_{\mathrm{fe}}^{1} V_{\mathrm{LF}, \mathrm{fe}}^{1}+K_{\mathrm{fe}}^{2} V_{\mathrm{LF}, \mathrm{fe}}^{2}+K_{\mathrm{fe}}^{3} V_{\mathrm{LF}, \mathrm{fe}}^{3}\right) \mathrm{e}^{-\mathrm{j} \beta \ell} \\
\left.V_{\mathrm{ne}}\right|_{\Gamma_{\mathrm{ne}}=0} & =\left(K_{\mathrm{ne}}^{1} V_{\mathrm{LF}, \mathrm{ne}}^{1}+K_{\mathrm{ne}}^{2} V_{\mathrm{LF}, \mathrm{ne}}^{2}+K_{\mathrm{ne}}^{3} V_{\mathrm{LF}, \mathrm{ne}}^{3}\right),
\end{aligned}
$$

where $K_{\mathrm{fe}}^{u}$ is the far-end correction factor of the $u$ th line segment. $V_{\mathrm{LF}, \mathrm{fe}}^{u}\left(V_{\mathrm{LF}, \mathrm{ne}}^{u}\right)$ denotes the the low-frequency far-end (near-end) voltage of the $u$ th segment according to (1). $\ell$ is a shorthand for the line's total length $\ell_{1}+\ell_{2}+\ell_{3}$.

The correction factors $K^{u}$ are found by integrating $i w^{*}$ along the line. Now that the line is no longer parallel with $z$ everywhere anymore, let $s$ denote the length along the line. For example,

$$
K^{2}=\frac{1}{\ell_{2}} \int_{\ell_{1}}^{\ell_{1}+\ell_{2}} i(s) w^{*}(s) \mathrm{d} s=\frac{1}{\ell_{2}} \int_{\ell_{1}}^{\ell_{1}+\ell_{2}} \mathrm{e}^{-\mathrm{j} k_{z} \ell_{1}} \mathrm{e}^{\mp \mathrm{j} \beta s} \mathrm{~d} s,
$$

because the incident field is uniform along segment 2. In general,

$$
K^{u}=\frac{1}{\mathrm{j}\left(-k_{s} \mp \beta\right) \ell_{u}}\left(\mathrm{e}^{\mathrm{j}\left(-k_{z} z_{\text {end }} \mp \beta s_{\text {end }}\right)}-\mathrm{e}^{\mathrm{j}\left(-k_{z} z_{\text {begin }} \mp \beta s_{\text {begin }}\right)}\right),
$$

where $k_{s}$ is the incident wave vector along the line segment and $k_{z}$ is the incident wave number.

Likewise, for the low-frequency contributions $V_{\mathrm{LF}}^{u}$, the field orientation with respect to the line segment must be taken into
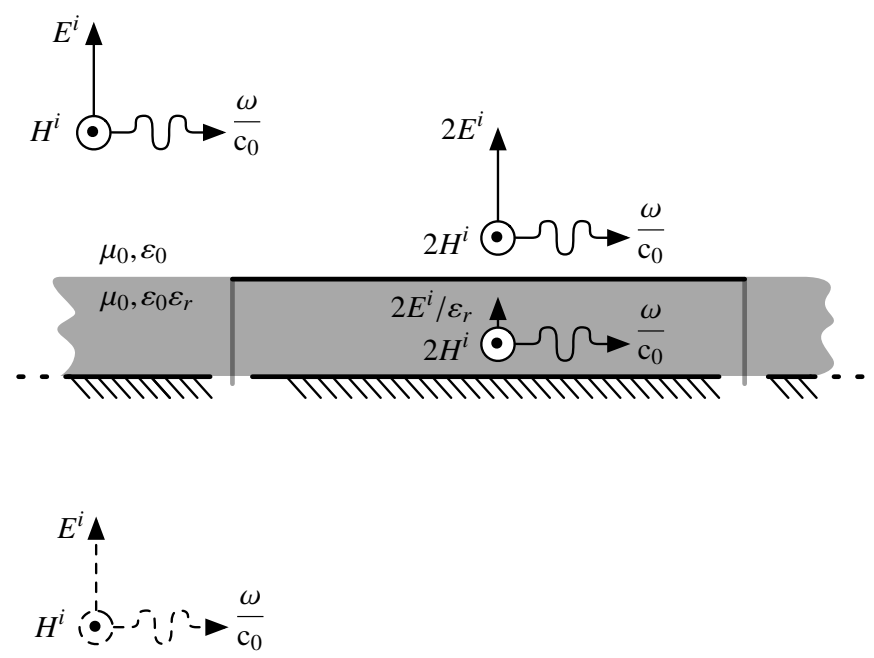

Figure 7. The far-away plane wave source (top left) is reflected by the microstrip's ground plane (image source at the bottom left). This results in the fields shown at the right. 
account. For example,

$$
V_{\mathrm{LF}}^{2}=-\mathrm{j} \omega c \frac{E^{i}}{\varepsilon_{r}} Z_{c} h \ell_{2}=-\mathrm{j} \frac{\omega}{\mathrm{c}_{0}} E^{i} h \ell_{2} \frac{\sqrt{\varepsilon_{r, \mathrm{eff}}}}{\varepsilon_{r}},
$$

because the incident magnetic field is parallel to line segment 2 , hence it does not contribute to the terminal voltage.

Because the GTEM-cell has a $50 \Omega$ input impedance and the far end is terminated in $50 \Omega$, the $V_{\text {fe }} / V_{\text {septum }}$ ratio equals the transfer $S_{21}$ from septum to far end. A Python script took $3 \mathrm{~ms}$ on a Intel $2.53 \mathrm{GHz}$ Core 2 Duo processor to calculate these far-end induced voltages according to (5). The code can be viewed and run online [8], in comparison with the measurement data.

\section{Full-WAVE SOLVER}

The trace was entered in CST Microwave Studio as an infinitely-thin, perfect electric conductor (PEC). The ground plane was a $75 \times 75 \mathrm{~mm}$ PEC beneath the trace. The substrate was a lossless dielectric layer with $\varepsilon_{r}=4.6$, covering the ground plane. The trace was terminated in $50 \Omega$ discrete face ports. The geometry was meshed using a hexahedral $\lambda / 10$ mesh for $20 \mathrm{GHz}$, except near the microstrip: one mesh cell across the dielectric thickness and at least two mesh cells across the trace width. The Perfect Boundary Approximation (PBA) compensates for the staircase meshing of the mitered trace corners.

All boundary conditions were set to 'open and add space': that is, $\lambda / 8$ extra space was added, terminated by 4 perfectly matched layers (PMLs). This geometry was then illuminated with a vertically polarized plane wave excitation, as illustrated in Figure 8a. The plane wave electric field strength was $1 / d$, where $d$ is the GTEM-cell septum distance.

In order to take the terminal impedances into account, a geometry-circuit co-simulation was chosen, also because of its scaleability to many and non-linear loads. Hence, the above mentioned geometry with plane wave excitation was entered as one schematic block in the co-simulation. At the near end, a lossless transmission line was connected, representing the coaxial connection with delay $\tau_{d}$ up to the short standard, followed by a perfect short circuit. At the far end, a perfect load was connected, monitored by a voltage probe. As the structure is illuminated with the field caused by $1 \mathrm{~V}$ at the septum, the output voltage in volts predicts the transfer $S_{21}$ from septum to far end. The resulting schematic is depicted in Figure 8b.

The inherent causality of co-simulation requires a timedomain solver and a Finite Integration Technique (FIT)-based solver [9] was chosen. The co-simulation was then run with a $-60 \mathrm{~dB}$ accuracy criterion on a $2 \times 2.5 \mathrm{GHz}$ Intel ${ }^{\circledR}$ E5 with 16 GB RAM and an NVIDIA ${ }^{\circledR}$ Tesla $^{\mathrm{TM}} 20$ graphics processing unit (GPU), which took $35 \mathrm{~s}$.

In order to allow for phase comparison, the phase reference of the plane wave had to be moved from the simulation boundary to the near end. Therefore, the simulated far-end voltage was advanced by the corresponding time of flight in

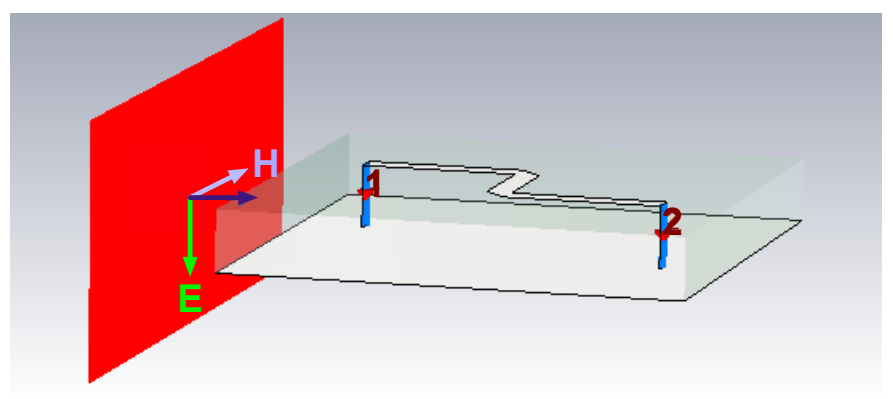

(a) Geometry (figure not to scale, in order to see the substrate) as entered into CST Microwave Studio. The dielectric substrate is shown translucent. Note the polarization of the incident plane wave from the left.

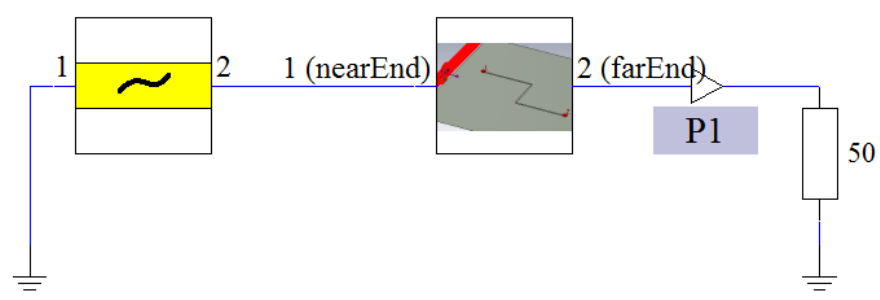

(b) Co-simulation schematic as entered into CST Design Studio. The first block is a lossless transmission line defined by its delay $\tau_{d}$, the second block is the geometry with excitation of Figure 8a.

Figure 8. Overview of the CST Studio full-wave simulation.

the comparison script, of which the results are compared with the other methods in Figure 11.

The log-frequency average difference between simulation and the extended Taylor prediction, a measure for bias, was $+0.8 \mathrm{~dB}$ over the $50 \mathrm{MHz}$ to $20 \mathrm{GHz}$ range. The average absolute error was $1.4 \mathrm{~dB}$. Decreasing the accuracy criterion to $-80 \mathrm{~dB}$ barely changed the simulation results, so the $-60 \mathrm{~dB}$ accuracy criterion was kept. Decreasing the mesh size to $\lambda / 20$ slightly improved the results: $+0.4 \mathrm{~dB}$ average error and $0.9 \mathrm{~dB}$ average absolute error. Because this longer simulation yielded little improvement, the first simulation will be used in all comparisons.

\section{EXPERIMENT}

This section describes the measurement set-up in more detail, discusses its quality and describes the measurement itself.

\section{A. Set-up Details}

In order to fabricate the case study with small and known mechanical uncertainties, the PCB was made in a four-layer industrial FR4 process, with mitered bends, traces matched to $50 \Omega$ and connector-to-board transitions optimized, as can be seen in Figure 9. The PCB's ground contact was matched to the shape of the GTEM-cell aperture to smooth the transition from the ground plane to the GTEM-cell wall. The resulting PCB is shown in Figure 10. In order to express the layout as an objective function of fabrication and experiment constraints, it was described in code with PyPCB [10].

To numerically predict the measurements, some set-up specific numbers had to be entered into both the analytical model 


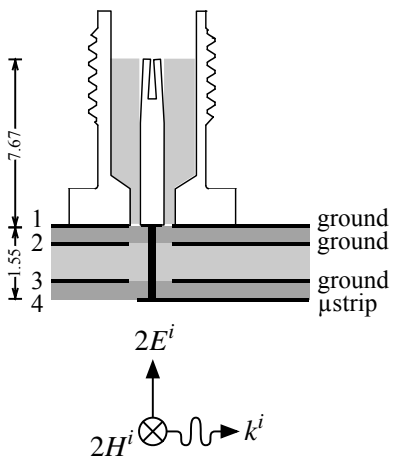

(a) Lateral cross-section.

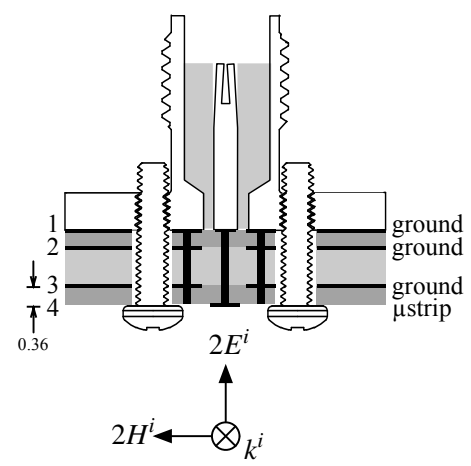

(b) Frontal cross-section. The M2 screws are made of nylon.

Figure 9. SMA-to-microstrip launch: a Molex SD-73251-185 SMA jack screwed onto a footprint designed using CST to minimize reflections (drawing not to scale, dimensions in $\mathrm{mm}$ ).

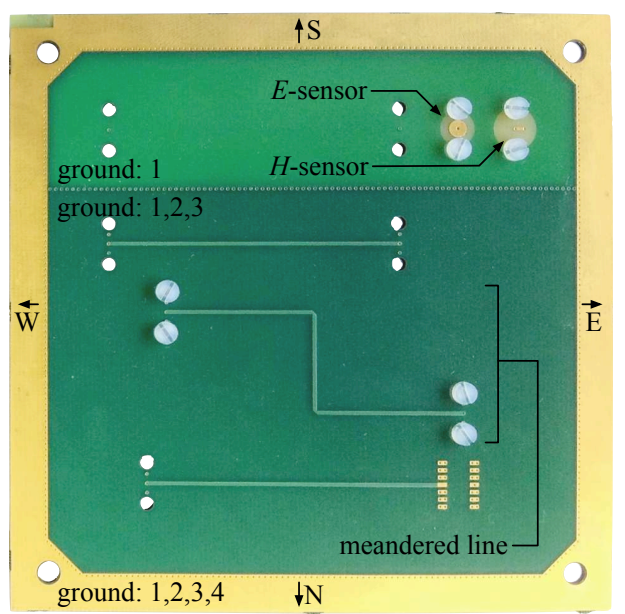

Figure 10. Bottom side (layer 4) of the GTEM $10 \times 10 \mathrm{~cm} \mathrm{PCB}$. Only the features used in this paper are labelled. Field sensors allow to gauge the field homogeneity.

and the full-wave simulation. The GTEM septum distance $d$ was measured to be $42.2 \mathrm{~mm}$ on average under the PCB aperture. From the near end of the trace to the short circuit, the signal traverses the PCB, the SMA connector and the short standard. The PCB has a thickness $t$ of $1.55 \mathrm{~mm}$ and a relative permittivity $\varepsilon_{r}$ of 4.6. Using Agilent's ADS, the filling factor of the microstrip was calculated to be 0.67 on average over the frequency range of interest. With this approximation, the microstrip's effective relative permittivity $\varepsilon_{r \text {,eff }}$ was calculated to be 3.4. Modeling this path as a homogeneous waveguide, the delay $\sqrt{\varepsilon_{r}} \cdot t / \mathrm{c}_{0}$ should approximate $11.1 \mathrm{ps}$. The SMA connector has a Teflon dielectric $\left(\varepsilon_{r}=2.1\right)$ of $7.67 \mathrm{~mm}$ from board to reference plane. Similarly, this should account for 37.1 ps. The Agilent 85052D-60006 short circuit is specified to have a $31.78 \mathrm{ps}$ delay, totaling to a delay $\tau_{d}$ of $79.95 \mathrm{ps}$.

\section{B. GTEM Field Uniformity}

Before illuminating the PCB with the GTEM cell, the field uniformity needs to be assessed. Therefore, $E$-field and $H$ -
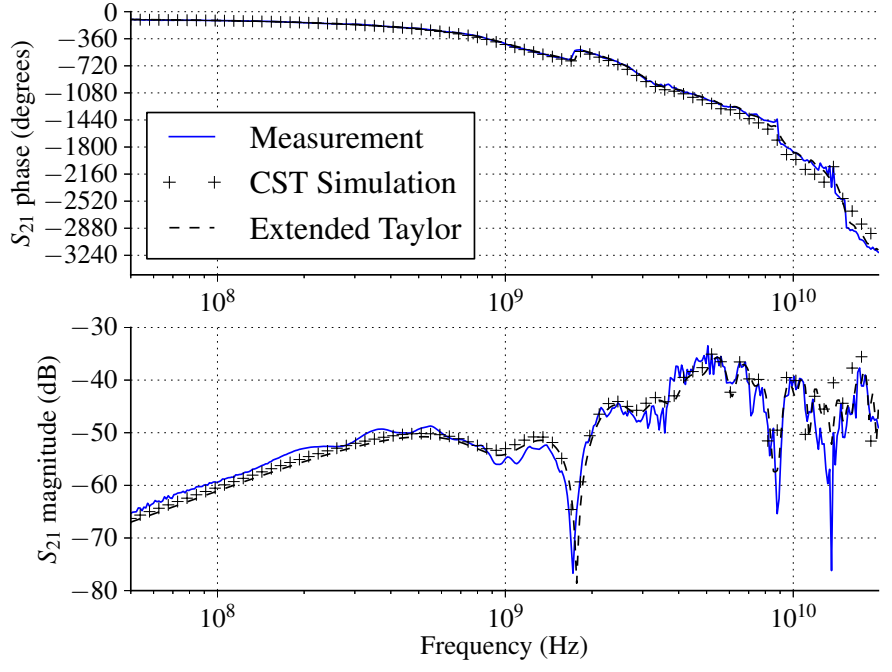

Figure 11. Field-to-trace coupling of the meandered trace, illuminated in a GTEM cell, as measured, full-wave simulated and calculated with the extended Taylor model (Equation 5). Port 1 is the GTEM cell input, port 2 is the trace's far end, while the near-end is terminated in a short circuit. This figure can be reproduced online [8].

field sensors were integrated in one corner of the PCB. The $H$-field sensor consists of two vias and a trace that form a loop tangential to the septum, as short as technologically possible for the field to be uniform in the loop $(1.23 \mathrm{~mm})$, as high as technologically possible to increase its surface $(1.50 \mathrm{~mm})$. To measure the $E$-field, a disk of $3 \mathrm{~mm}$ diameter was used, in order to stay under $\lambda / 10$ at $10 \mathrm{GHz}$ and to have sufficient coupling surface to measure strong enough a signal.

The transfer from GTEM cell septum to the $E$-field sensor was measured at four different $\mathrm{PCB}$ orientations by rotating the PCB. Up to $5 \mathrm{GHz}$, the log-frequency averaged difference between the maximum and minimum transfer was $1.4 \mathrm{~dB}$, and $5.8 \mathrm{~dB}$ between $5-20 \mathrm{GHz}$. As far as the $H$-field sensor is concerned, the average difference was $1.5 \mathrm{~dB}$ up to $5 \mathrm{GHz}$, and $3.9 \mathrm{~dB}$ between $5-20 \mathrm{GHz}$, taking into account both positions. These numbers suggest that the field non-uniformity is in the order of a few $\mathrm{dB}$.

\section{Measurements}

The measurement of Figure 1 was performed, yielding the $S_{21}$ plotted in Figure 11. The log-frequency average difference between measurement and extended Taylor prediction was $+0.2 \mathrm{~dB}$ over the $50 \mathrm{MHz}$ to $20 \mathrm{GHz}$ range. The average absolute error was $2.2 \mathrm{~dB}$.

The ripple in the measurement is supposedly caused by standing waves in the GTEM-cell. However, no satisfactory explanation was found for the low-frequency offset of $+2 \mathrm{~dB}$ with respect to both the modified Taylor model and the fullwave simulation; explanations in the literature deal with zerocentered deviations from the $V_{\text {septum }} / d$ estimator. In general, it should be noted that quite some imperfections of the measurement set-up were not taken into account in model and simulation: the substrate permittivity being frequencydependent, skin loss and dielectric losses in the traces, the 
discontinuity between the GTEM-cell wall and the substrate, and the non-uniformity of the illuminating field.

\section{CONCLUSIONS AND PERsPectives}

The coupling of an incident grazing plane wave to the farend of a meandered, near-end shorted PCB trace with ground plane was studied. To that end, the modified Taylor model was extended to include a near-end non-characteristic load. This extension should hold for any electrically long, lossless transmission line, illuminated by a linearly polarized plane wave and terminated characteristically at its far end. The solution was still closed-form, so the calculation time was only $3 \mathrm{~ms}$.

Then, a full-wave electromagnetic solver was compared against this model. In CST, an infinitely-thin, perfectlyconducting microstrip trace with a lossless dielectric was illuminated with a far-field source. Using time-domain cosimulation, the near-end short circuit was taken into account. The solution differed by $+0.8 \mathrm{~dB}$ from the extended Taylor prediction, averaged over log-frequency in the $50 \mathrm{MHz}$ to $20 \mathrm{GHz}$ band. The average absolute error was $1.4 \mathrm{~dB}$. The simulation time was $35 \mathrm{~s}$.

Finally, a measurement in a GTEM cell was performed and compared against the extended Taylor model. The average difference with the extended Taylor model was $+0.2 \mathrm{~dB}$ with an average absolute error of $2.2 \mathrm{~dB}$. This error is in the order of the field strength variation, as measured with integrated $H$ - and $E$-field sensors. As the correlation with measurement was already quite good, dielectric losses, amongst others, were not incorporated in the full-wave simulation neither in the modified Taylor model.

To sum up, the extended Taylor model, despite its many simplifying assumptions, is able to quickly estimate the field-totrace coupling for grazing incidence and one non-characteristic load.

The next step would be to adapt the modified Taylor model to approach the near-field illuminated suspended wire of IEEE benchmark case 5.3.1.2 [11]. This requires to take into account a far-end non-characteristic load $(1 \mathrm{k} \Omega)$, which would introduce the complexity of bidirectional reflections and require another measurement method. For industrial applicability, nongrazing, arbitrary polarized incidence should be taken into account as well, probably using worst-case analysis to simplify the solution. Finally, the solution should be made accessible to PCB designers, to estimate PCB immunity quickly and early in the design process.

\section{ACKNOWLEDGEMENTS}

This research project was co-financed by the French national project SEISME (simulation of emissions and immunity of electronic systems). We thank Mohamed Latrach for his effort to provide us with state-of-the-art tools and equipment.

\section{REFERENCES}

[1] C. R. Paul, Introduction to Electromagnetic Compatibility. Hoboken, New Jersey: Wiley, 2006.
[2] J. L. Lagos and F. L. Fiori, "Worst-case induced disturbances in digital and analog interchip interconnects by an external electromagnetic plane wave - part I: modeling and algorithm", Electromagnetic Compatibility, IEEE Transactions on, vol. 53, no. 1, pp. 178-184, Feb. 2011. DOI: 10.1109/TEMC.2010.2085005.

[3] M. Magdowski and R. Vick, "Closed-form formulas for the stochastic electromagnetic field coupling to a transmission line with arbitrary loads", Electromagnetic Compatibility, IEEE Transactions on, vol. 54, no. 5, pp. 1147-1152, Oct. 2012. DOI: 10.1109/TEMC.2012.2193130.

[4] M. Leone and H. L. Singer, "On the coupling of an external electromagnetic field to a printed circuit board trace", Electromagnetic Compatibility, IEEE Transactions on, vol. 41, no. 4, pp. 418-424, Nov. 1999. DOI: 10.1109/15.809842.

[5] S. T. Op 't Land, M. Ramdani, R. Perdriau, M. Leone, and M. Drissi, "Simple, Taylor-based worst-case model for field-to-line coupling", JPIER, vol. 140, pp. 297-311, Jun. 2013. DOI: 10.2528 / PIER13041207.

[6] S. T. Op 't Land, T. Mandić, M. Ramdani, A. Barić, R. Perdriau, and B. Nauwelaers, "Comparison of field-to-line coupling models: coupled transmission lines model versus single-cell corrected Taylor model", in Electromagnetic Compatibility (EMC EUROPE), 2013 International Symposium on, Sep. 2013, pp. 276-281.

[7] IEC 62132-2 integrated circuits - measurement of electromagnetic immunity $150 \mathrm{kHz}$ to $1 \mathrm{GHz}$ - part 2: measurement of radiated immunity - TEM-cell method, Jul. 2004.

[8] S. T. Op 't Land, Extended Taylor online code and measurements for a meandered microstrip, May 2014. [Online]. Available: http://www. runshare.org/CompanionSite/site.do?siteId=369.

[9] T. Weiland, "A discretization model for the solution of Maxwell's equations for six-component fields", Archiv Elektronik und Übertragungstechnik, vol. 31, pp. 116-120, 1977.

[10] PyPCB - specifying PCB layout in Python, Nov. 2012. [Online]. Available: http://github.com/eseo-emc/pypcb/wiki.

[11] IEEE recommended practice for validation of computational electromagnetics computer modeling and simulations, 2010. DOI: 10.1109/ IEEESTD.2011.5721917 